Original Research Paper

\title{
Effects of Visible and Near Infrared Polarized Lights on Spoofing Face Detection
}

\author{
Azim Zaliha Abd Aziz \\ Faculty of Informatics and Computing, Universiti Sultan Zainal Abidin, \\ Tembila Campus, 22200 Besut, Terengganu, Malaysia
}

\author{
Article history \\ Received:24-12-2018 \\ Revised: 13-01-2018 \\ Accepted: 21-02-2019 \\ E-mail: azimzaliha@unisza.edu.my
}

\begin{abstract}
Face spoofing countermeasure is vital to avoid an imposter from gaining access to security biometric systems by using face masks in various forms that mimic a valid user face. Recently, several studies have shown the ability of visible polarized light in distinguishing real and fake faces. In this paper, polarization imaging systems using visible and near infrared (NIR) are proposed to examine the effects on the polarization images as trial to distinguish between genuine face and spoof face presentation attacks: photo paper and iPad face display; based on the optical properties of the materials. The findings from the investigations suggest that in general, NIR light could not be used to distinguish between genuine face and photo paper under a polarization lighting condition. In contrast, the visible light provides significant difference of the Stokes images between the materials. Classification between real face and iPad display can easily be done by manipulating the polarization angle. A new feature fusion formula named as the SDOLP3F is introduced to differentiate between the real and the fake traits. The SDOLP3F results presented in this paper show the highest accuracy rate compared to the individual measures. The results illustrate the robustness of the proposed anti-spoofing algorithm based on a small sample.
\end{abstract}

Keywords: Anti-Spoofing, Face Biometric, Polarized Light, Polarization Imaging System, Spoofing Attacks

\section{Introduction}

Biometric application refers to the automated recognition of individuals based on their physiological and/or behavioural characteristics (Jain et al., 2004). Examples of biometric characteristics are Deoxyribonucleic Acid (DNA), ear, face, fingerprint, gait, iris, keystroke, odour, palmprint, retinal scan, signature and voice. Due to the high demand on these biometric applications, fingerprint, face and iris have been the three most popular and mature modalities among the others (Jain et al., 2004). Moreover, the availability of large fingerprint, iris and face databases, which have been collected by various agencies all over the world, has also led to the demand. However, the resistance of this rapidly emerging technology to external attacks has become a critical issue.

In particular, face spoofing is an attack where photograph, video or mask of a valid user is presented in front of face recognition system as trial to gain access. From the observation, Face Recognition (FR) systems are vulnerable to spoofing attacks (Kose and Dugelay, 2013a). This vulnerability has attracted the attention of researchers in the biometric community to work on countermeasures to detect spoof representation attacks. For instance, an imposter can obtain a photo of an authorized person, plays a video, or display a 3D model such as a face mask which mimics a valid individual, in front of the sensor to gain access (Pan et al., 2008).

Among the spoofing methods, the most common types being used are the printed photo paper and the video attacks. These two representation attacks are popular due to the low-cost, convenience and also can be easily acquired. The existing spoofing countermeasures are based on several types of approaches: Liveness detection, motion analysis, texture analysis and reflectance based analysis. Liveness detection which is 
based on eyes blinking and lips movement has been tricked with perforated mask in the eyes and mouth. The motion based counter spoofing has been challenged with recorded video playback in front of the camera. The third approach aims to detect real face by analyzing the texture of the face image to find spoofing clues such as printing artifacts (Bai et al., 2010) or blurring (Li et al., 2004). Finally, the reflectance characteristics of real face and fake traits are analyzed as spoofing face detection.

Besides the different types of countermeasure-based analyses, the main key to differentiate between a genuine human face and other representation attacks is to manipulate the physical and optical properties of the skin. Physically, skin consists of multi layers structure and due to these various layers, skin produces more than one reflections: Surface and subsurface reflections. Polarization imaging technique is one of the methods that could be used to distinguish between the two reflections (Matsubara, 2012; Jacques et al., 2000; Bae et al., 2010; Philp et al., 1987). In addition, the degree of polarization of each polarized image can also be used as the classification parameter. The Stokes parameters and the Degree Of Polarization (DOP) are two parameters applied to differentiate between materials (Zallat et al., 2003; Mahendru and Sarkar, 2012; Wolff and Boult, 1989; Hua and Wolff, 1996; Wolff, 1990; Sarkar et al., 2011). A study by (Abd, 2017) has demonstrated the ability of visible polarized light to differentiate between real face and photo paper based on the Stokes parameters.

Motivated by the previous findings of using polarized light to differentiate between materials, we adopted the similar approach as trial to distinguish between real human face and fake faces used to spoof a biometric system. In this study, we would analyse the effect of using different polarized light sources on the polarized reflection among the materials. Besides using the face-paper polarized dataset introduced by (Abd, 2017), we also captured images under the visible and NIR polarized lights. Additionally, an iPad representation attack is added to this dataset. At the end of this paper, we introduce our newly developed classification fusion formula known as the SDOLP3F.

The paper is organized as follows. Section 2 includes related work on the existing countermeasures of the spoofing attacks. In Section 3, the introduction to the human skin structure and the parameters used throughout this paper are discussed. In Section 4, we present our polarization imaging system and the selfcollected dataset. The proposed method for face spoofing detection is described in Section 5. In Section 6, experimental results are presented and discussed. The newly proposed fusion approach is presented in Section 7. Finally, conclusion is drawn in Section 8.

\section{Related Work}

Face spoofing attacks can be generally classified into 2D and 3D attacks. For instance, paper photo and iPad representation attack are $2 \mathrm{D}$ attacks while a $3 \mathrm{D}$ face mask is $3 \mathrm{D}$ attack. Due to these types of attacks, there have been various countermeasures to stop an imposter from gaining access to biometric security systems. Texture based approaches as in (Jukka et al., 2011; Erdogmus and Marcel, 2013) rely on Local Binary Pattern (LBP) to analyse the micro-texture patterns of both real face and fake face images. The approach is then used for further exploration amongst 3D facial mask compared to a planar printing mask (Kose and Dugelay, 2013b). LBP has shown convincing classification results.

The fact that each material has its own reflection properties has burst the other side of face counter spoofing analysis. The countermeasure based on reflectance information has attracted more researcher attention (Kose and Dugelay, 2013b; Zhang et al., 2011; Kim et al., 2009). Kose and Dugelay (2013b), the authors proposed a reflectance based analysis on the texture images from a $2 \mathrm{D}+3 \mathrm{D}$ face mask. The images were decomposed into illumination and reflectance components by using variational retinex algorithm. This proposed method has been proved to have better features information compared to texture analysis for the purpose of mask detection.

Another study presents a multispectral reflectance using multispectral lighting to discriminate between a real face, a photo face and variety of face masks made of plastic, silica gel, paper, plaster and sponge (Zhang et al., 2011). As a result, two discriminative light wavelengths ( 850 and $1450 \mathrm{~nm}$ ) have been chosen. In (Kim et al., 2009), the reflection properties of a facial skin and mask materials are analysed under two discriminative wavelengths $(685$ and $850 \mathrm{~nm})$. However, the analyses were conducted on a variety of materials physically not a mask that mimic the real faces.

During the past 20 years, polarized light has been used to classify materials. A similar series of experiments are proposed, which claim that polarized reflection can be used to distinguish between metal and dielectrics objects (Wolff, 1989). (Later Sarkar et al., 2011), presented a real-time CMOS image sensor to differentiate between metal and dielectric surfaces, claiming that polarization of the reflected component varies with the conductivity of the metallic surface. The Fresnel reflection coefficients and the degree of polarization were used to measure the variations of reflection.

After the success of the polarization imaging technique to classify between materials, a considerable amount of literature has been published on polarized reflection. In 2007, a polarization imaging device is demonstrated to measure the Stokes components in order 
to distinguish between breast tissue in health or with cancer (Bin et al., 2007). In the study by (Mahendru and Sarkar, 2012), the polarized reflection is examined to classify among transparent and opaque objects.

Recently, a study by (Rudd et al., 2016), proposed an approach in which polarized light has been used as trials to differentiate between a genuine face, LCD screen and paper mask. They have shown the differences of the polarization images between the real face, LCD and paper mask. However, the images have not yet been analysed by any parameters. Therefore (Abd, 2017) proposed the Stokes parameters which have been able to distinguish between real face and photo paper. The mean, standard deviation and kurtosis of the data distribution between the two materials have shown high True Positive Rate (TPR) and low False Positive Rate (FPR). However, the classification accuracy result based on a single parameter would be limited.

Hence, we proposed a method of feature fusion formula known as the SDOLP3F for better detection accuracy. The novelties of our study can be listed as follows:

- The newly proposed classification fusion formula is based on polarized reflectance analysis to differentiate between real and fake traits. This SDOLP3F algorithm has achieved a high detection accuracy rate and could be used to detect spoofing attacks under visible polarized light

- In has been proved that the statistical measures could be used to detect photo paper under visible polarized light (Abd, 2017). In this study, the statistical measures are fused with density of the distribution mode for better classification accuracy

- To the best our knowledge, there is no study on the spoofing detection under polarized NIR light. In this paper, the experimental results show that polarized NIR light could not be used to distinguish between real face and photo paper

- Previous studies on spoofing countermeasure against live video attacks have proposed motionbased technique. However, by using the proposed polarization imaging system, we have successfully blocked any trial to impersonate a valid individual by using an iPad display

\section{Theories Supporting the Studies}

\subsection{Physical Introduction to Human Skin}

Skin consists of various layers structure. Some analysts have listed three layers structure of human skin which are epidermis, dermis and fat layer (SoLing and Ling, 2001). The first layer is called epidermis which is the outermost layer of the skin. Epidermis layer contains particles called melanin which act as absorption and scattering agents. The quantity of melanin in the epidermis determines the skin color of a human. Dermis is a thick layer underneath the epidermis layer which contains properties such as hemoglobin and collagen. The third layer is the deepest layer made of fat and connective tissue, known as subcutaneous tissue.

\subsection{Polarization and Interaction with Human Skin}

Normal light wave from light source such as table light, vibrates in multi directions until it lies on a surface. This multi-directional light wave is also known as unpolarized light. Polarization is a process where unpolarized light turns to polarized light. One of the methods to produce polarized light is by using a device known as polarizer. Polarizer is an optical device that allows unpolarized light wave to transfer through only in one direction to become polarized light.

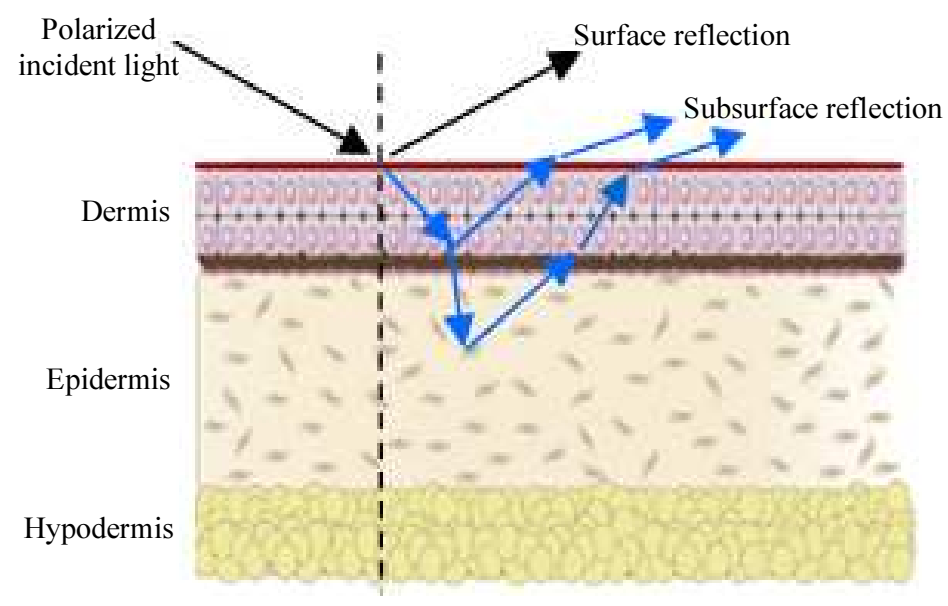

Fig. 1: The interaction between polarized light and human skin (Picture of the skin) 
Figure 1 illustrates the interaction between polarized light and skin surface. Once the incident light hits the skin layer, part of the light is reflected by the flat oily cells on top of the dermis. This reflection is known as surface reflection. The remaining light that is not reflected, is then transmitted through the dermis to the epi-dermis layers. This transmitted light is refracted and absorbed by the melanin, collagen and hemoglobin within the skin layers before re-emerged back to the air through the skin surface. This second reflection is known as subsurface reflection.

Driven by these multi-reflections produced by human skin, polarization imaging system could be applied for further analysis on each reflection. In addition, the state of polarization of reflected light can be described by using the Stokes parameters. Theoretically, reflection properties are different between materials which lead to this study.

\subsection{The Stokes Parameters}

Light is made up of photons, which are packets of electromagnetic wave. The electromagnetic wave is said to be linearly polarized if the oscillation of the wave is confined to specific directions. Otherwise, the light wave is said to be unpolarized. Polarized light can be obtained by several ways such as absorption, reflection, refraction and scattering. Other than that, a polarizer is used to obtain polarized light by mounting the polarizer sheet in front of an unpolarized light source.

The state of polarization of reflected light wave can be described by the Stokes parameters. These parameters were introduced by Sir G. C. Stokes in 1852. The parameters have four components denoted as $S_{0}, S_{1}, S_{2}$ and $S_{3}$. These components are presented in a $4 \times 1$ column matrix known as the Stokes vector, as shown as:

Stokes $=\left[\begin{array}{l}S_{0} \\ S_{1} \\ S_{2} \\ S_{3}\end{array}\right]$

The Stokes parameters represented in (1) can be described as intensity $\left(S_{0}\right)$, degree of polarization $\left(S_{1}\right)$, plane of polarization $\left(S_{2}\right)$ and ellipticity $\left(S_{3}\right)$. Since only linear polarization is used in this study, circular and elliptical polarization does not occur. Therefore, the component $S_{3}$ which relates to the circular polarization is omitted. The components $S_{0}, S_{1}$ and $S_{2}$ are obtained using images captured at four polarization angles as:

$$
\begin{aligned}
& S_{0}=\operatorname{Im} g_{0}+\operatorname{Im} g_{90} \\
& S_{1}=\operatorname{Im} g_{0}-\operatorname{Im} g_{90} \\
& S_{2}=\operatorname{Im} g_{135}-\operatorname{Im} g_{45}
\end{aligned}
$$

where, $I m g_{0}, I m g_{45}, I m g_{90}$ and $I m g_{135}$ are the polarized images captured at polarization angles of $0^{\circ}, 45^{\circ}, 90^{\circ}$ and $135^{\circ}$, respectively. Polarizer angles are referred to the angle of the polarizer $\mathrm{P}_{1}$ which is mounted in front of the camera lens. Once the Stokes parameters have been derived, they are then used to generate the final image known as the Stokes Degree of Linear Polarization Image (SDOLP) as:

$$
I_{S D O L P}=\frac{\sqrt{S_{1}^{2}+S_{2}^{2}}}{S_{0}}
$$

An incident light that reflected from a surface or the light that is transmitted through a polarizer is partially polarized (Sarkar et al., 2011). The Degree Of Polarization (DOP) is used to measure the portion of reflected light which is polarized compared to the total amount of the reflected light. The DOP is also denoted as partial polarization. The scalar value of the DOP is between 0 and 1 . The DOP can also be expressed in terms of maximum and minimum light intensities transmitted through the linear polarizer as:

$$
D O P=\frac{I_{\text {max }}(x, y)-I_{\text {min }}(x, y)}{I_{\text {max }}(x, y)+I_{\text {min }}(x, y)}
$$

where, $I_{\max }$ is the maximum light intensity while $I_{\min }$ is the minimum light intensity transmitted through the polarizer. Details explanation of setting the $I_{\min }$ and $I_{\max }$ is discussed in the next section. Thus, let $I_{\max }$ be an image taken under a $0^{\circ}$ polarization and Imin be an image captured under a $90^{\circ}$ polarization. The two images are denoted as $I m g_{0}$ and $I m g_{90}$, respectively, which are then used to generate third image known as polarization image, $I m g_{\text {pol }}$. Hence, Equation (4) can be rewritten as:

$I_{p o l}=\frac{\operatorname{Im} g_{0}-\operatorname{Im} g_{90}}{\operatorname{Im} g_{0}+\operatorname{Im} g_{90}}$

From Equation (5), it can be seen that the $I m g_{0^{-}}$ $I m g_{90}$ and the $I m g_{0}+I m g_{90}$ are equivalent to $S_{1}$ and $S_{0}$, respectively. Therefore, Equation (5) can also be written as:

$I_{p o l}=\frac{\operatorname{Im} g_{0}-\operatorname{Im} g_{90}}{\operatorname{Im} g_{0}+\operatorname{Im} g_{90}}=\frac{S_{1}}{S_{0}}$

\subsection{The Bimodality Coefficient}

In statistics, a bimodal distribution is a continuous probability distribution with two different modes. The Probability Density Function (PDF) of a bimodality distribution should consists two or more peaks. The Bimodality Coefficient (BC) is one of the methods 
used to estimate the modality of data distributions. In this paper, the $\mathrm{BC}$ is applied to measure the modality distribution of each polarization image. The $B C$ values range from 0 and 1 , with empirical values of $\mathrm{BC}>0.555$ suggesting bimodal distribution (Pfister et al., 2013). Otherwise, the distribution is classified as unimodal.

The computation of the $\mathrm{BC}$ requires three inputs: The sample size $n$; the skewness; and the kurtosis. The formulation of the BC is computed in MATLAB based on the sample-bias corrected equation proposed by (Pfister et al., 2013) as:

$$
B C=\frac{m_{3}^{2}+1}{m_{4}+3\left(\frac{(n-1)^{2}}{(n-2)(n-3)}\right)}
$$

where, $m_{3}=$ skewness $(x, 0) ; m_{4}=$ kurtosis $(x, 0)-3$; and $n=$ sample size. The $\times$ refers to the input image, $I_{\text {pol }}$ or $\mathrm{I}_{\mathrm{SDOLP}}$.

\section{The Proposed Methods for Face Spoofing Detection}

The proposed face anti-spoofing method is based on two types of input images: $I_{\text {pol }}$ images created using equation (6); and $\mathrm{I}_{\mathrm{SDOLP}}$ images which are generated by using Equation (3). Figure 3 shows the schematic diagram of the proposed face spoofing countermeasure. As can be seen from Fig. 3, two types of face attacks that resemble the real subjects are used as the attempts to cheat the proposed framework. Firstly, iPad screen was used to display faces of the legitimate users in front of the camera. After that, photo of the real user face that has been printed on an A4 matte paper, was placed in front of the camera as trials to gain access. Then, the genuine subjects were asked to stand in front of the sensor for the recording processes. Images of the genuine faces and the spoof presentation attacks are captured at four polarization angles as has been mentioned earlier. Each of the images are then going through several processes: Crop, align, resize and averaged. The processed polarized images of each polarization angle are used as the input images to generate one Stokes degree of linear polarization $\left(\mathrm{I}_{\mathrm{SDOLP}}\right)$ image and one polarization $\left(\mathrm{I}_{\mathrm{pol}}\right)$ image by using Equations (3) and (6), respectively.

The data distributions of each $\mathrm{I}_{\text {pol }}$ and $\mathrm{I}_{\text {SDOLP }}$ images are generated in histograms. Further analyses have been carried out by six different measures: The mean, standard deviation, skewness, kurtosis, bimodality coefficient and mode of the density plot.

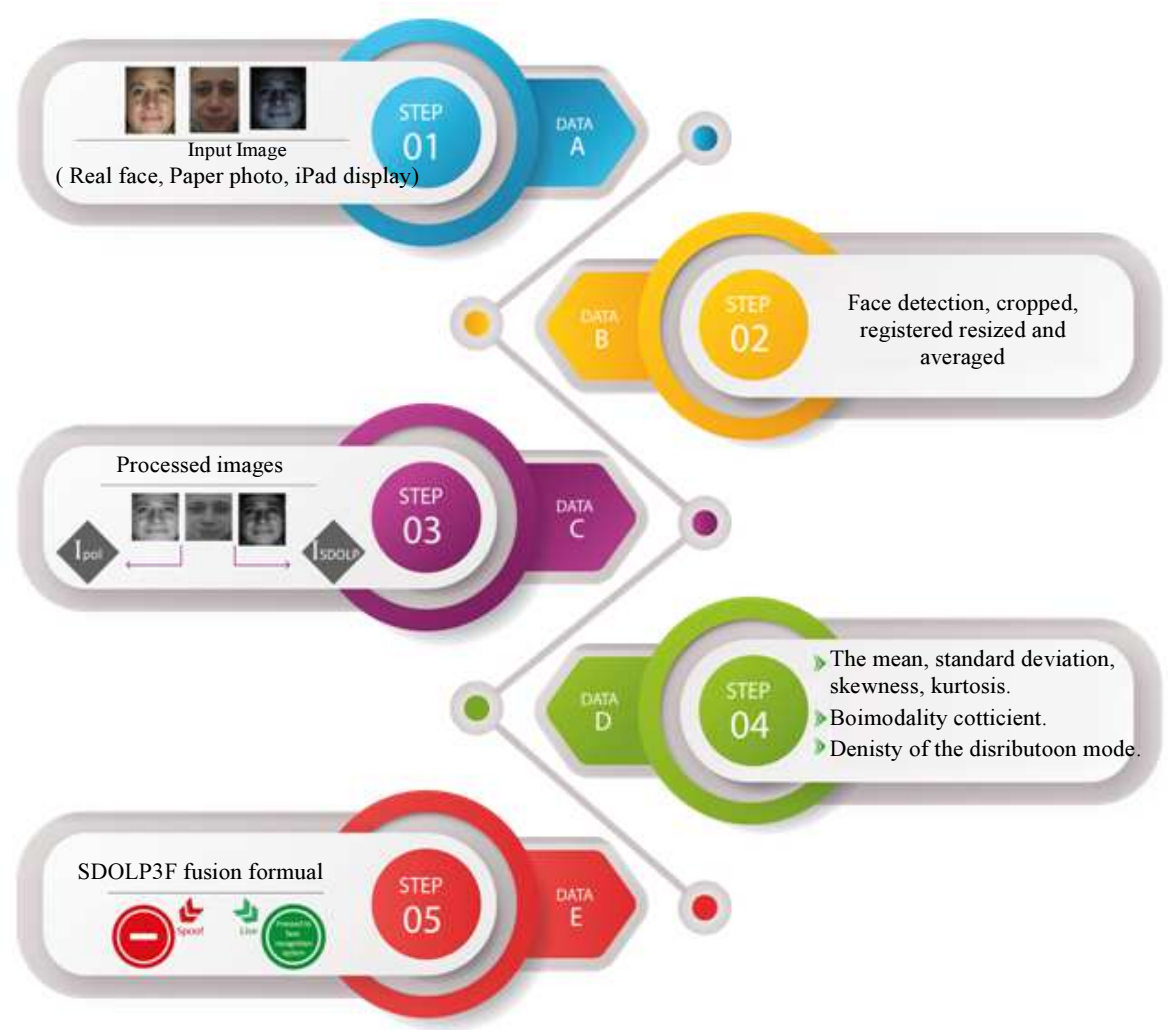

Fig. 3: The proposed anti-spoofing face detection system (framework) 
The scores of each measure are plotted to visualize the differences between materials. Then, a value which is considered as the most significant boundary between the materials is chosen as classification threshold.

Based on the threshold values, the types of materials are predicted by using the confusion matrix. For each measure, the TPR, the FPR and the classification accuracy rates are calculated. The measures with the highest fake (spoofing) face detection accuracy rates are then fed into the newly proposed face classification fusion formula named as the Stokes Degree Of Linear Polarization Fast Fusion Formula (SDOLP3F).

\section{The Polarization Imaging System}

\subsection{Experimental Setup}

Although two types of light sources with different wavelengths are used in this study, the experiment setup for each light is similar as illustrates in Fig. 4. The imaging system is similar to that in (Abd, 2017) which consists of a camera, two lights, two sheets of linear polarizer and one glass linear polarizer coupled with an angle rotator. The camera is placed $80 \mathrm{~cm}$ in front of the subject. A linear glass polarizer, $\mathrm{P}_{1}$ coupled with an angle rotator, is mounted in front of the camera lens. The angle rotator is used to adjust the $\mathrm{P}_{1}$ angle during the image recording process. Then, each of the light is placed $50 \mathrm{~cm}$ next to the right and left of the camera.
The lights are adjusted to illuminate the subject at an angle of $45^{\circ}$. The two linear polarizers denoted as $\mathrm{P}_{2}$ and $P_{3}$ are placed in front of each light. Both of the polarizer sheets are aligned in parallel to each other at the same polarization axis so that the lights wave travel in the same direction towards the subject. To check on the polarizer alignment, both of the polarizer sheets are placed back to back ideally in front of an object in order to view the object through the two sheets.

Figure 5 illustrates the steps to determine the polarization axis. Two polarizer sheets are placed next to each other. By slowly rotating the second sheet on top of the first sheet until all the light is blocked and the object can no longer be seen through the two sheets, the orientation of the polarizers is perpendicular to each other. Images captured at this position are labelled as $I_{\text {min }}$. The second polarizer is rotated once again until the object was clearly visible. At this orientation, the polarization axes of the polarizers are parallel and images captured under parallel polarization are denoted as $I_{\max }$. With this technique, the parallel orientation of the polarizers has been discovered and subsequently the polarizers are installed in front of each light source. The size of the polarizer sheets has to cover the whole light source. As in this study, the size of both polarizer sheets is $12 \times 12 \mathrm{~cm}$ which is sufficient to cover the entire table light source.

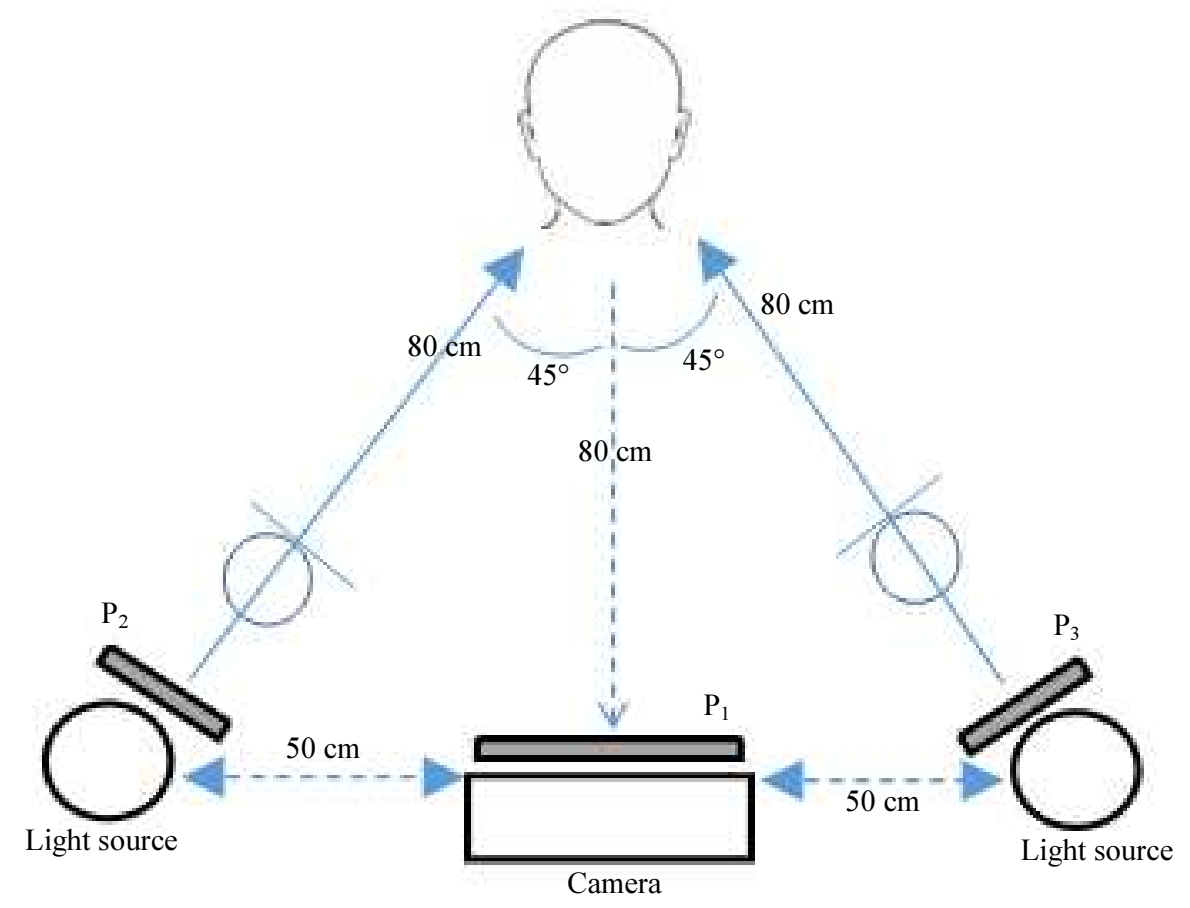

Fig. 4: The polarization imaging system 


\subsection{The Dataset}

To the best of our knowledge, no polarized spoofing image database is publicly available. In order to validate the effectiveness of the proposed method, a set of polarized images for both Visible (Vis) and NIR lights are captured by using the measurement setup shown in Fig. 4. Thirty people have been randomly selected as the subjects which consist of 11 women and 19 men of three different skin colours: Black, Caucasian and Asian. The recording is firstly carried out without any polarizer installed. This is to record images of the real faces which are then been printed on a paper and display on an iPad as spoofing attacks. Then, the $\mathrm{P}_{2}$ and $\mathrm{P}_{3}$ polarizers are mounted in parallel to each other in front of each light.

By manually rotating the angle of the glass linear polarizer, $\mathrm{P}_{1}$ to four polarization angles: $0^{\circ}, 45^{\circ}, 90^{\circ}$ and $135^{\circ}$, images of each subject are recorded at each of the angles. Noted that three frames of images are captured for all subjects at each $\mathrm{P}_{1}$ angles. The recording process has been carried out in a dark room to control the illumination. Hence, the intensity of the images captured is caused only by the polarized light source. The recorded images are cropped, resized, aligned and averaged which resulted in one image per subject at each $\mathrm{P}_{1}$ angle. A summary of the total images available in the Face-PaperiPad polarization dataset is presented in Table 1. The number of images in the dataset can be elaborated as:

- 37 subjects for genuine face and photo paper under visible light

- 8 iPad display attacks under visible light

- 24 subjects for genuine face, photo paper and iPad display under NIR light

- 4 polarization angles for each subject

- 3 frames for each polarization angle

- 1 processed image for each polarization angle of each subject

Table 1: The Face-Paper-iPad Polarization dataset

\begin{tabular}{llr}
\hline & \multicolumn{2}{l}{ Number of images } \\
& Visible light & NIR light \\
\hline Real face & 481 & 312 \\
Printed photo paper & 481 & 312 \\
iPad & 104 & 312 \\
\hline
\end{tabular}

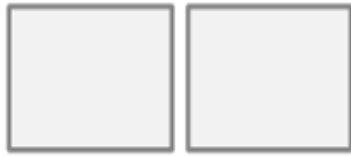

(a)

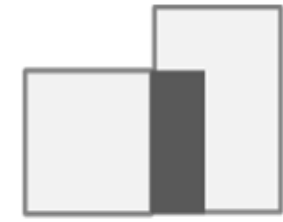

(b)

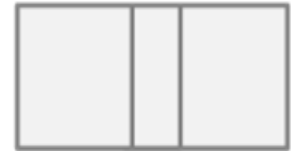

(c)

Fig. 5: The determination of the polarization axes for $\mathrm{P} 2$ and $\mathrm{P} 3$ polarizer sheets (a) the two polarizer sheets (b) perpendicular polarization; and (c) parallel polarization

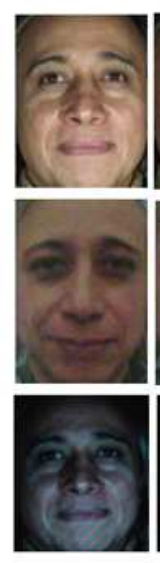

$0^{\circ}$
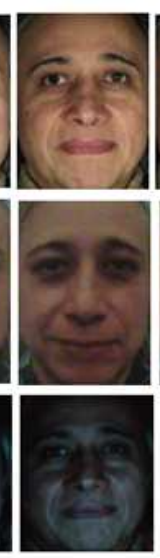

$45^{\circ}$
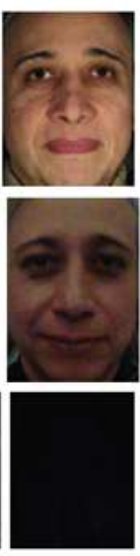

$90^{\circ}$
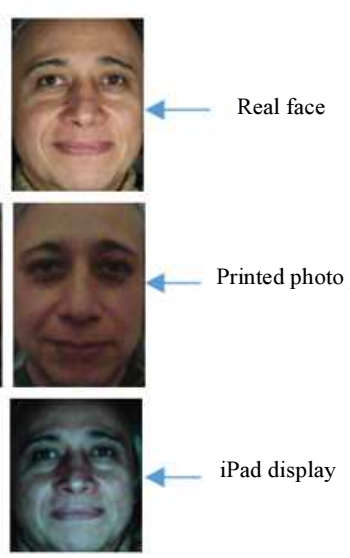

$135^{\circ}$

Visible light

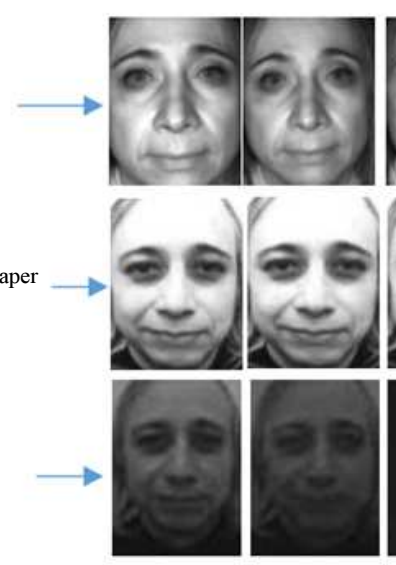

$452^{\circ}$
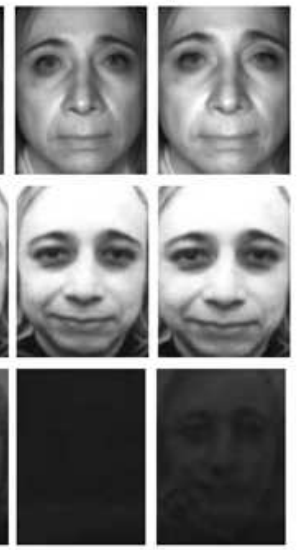

$90^{\circ}$

$135^{\circ}$

NIR light

Fig. 6: Sample of images captured at four polarization angles in the Face-Paper-iPad dataset. The first row is the real face, the second and the third rows are the printed photo paper and iPad display attacks 
For the purpose of analysis, images of one subject are presented throughout this paper. Figure 6 shows the RGB images of one subject recorded at four polarization angles: $0^{\circ}, 45^{\circ}, 90^{\circ}$ and $135^{\circ}$ under visible and NIR lights.

\section{Experimental Results}

The proposed spoofing countermeasure analyse the $\mathrm{I}_{\mathrm{pol}}$ and $\mathrm{I}_{\mathrm{SDOLP}}$ images of the genuine face, the printed photo paper and the iPad display. The $\mathrm{I}_{\text {pol }}$ and $\mathrm{I}_{\mathrm{SDOLP}}$ images are presented in Fig. 7 together with the Stokes components: $S_{0}, S_{1}$ and $S_{2}$.

\subsection{Detection Using the Mean, Standard Deviation, Skewness and Kurtosis}

As can be seen in Fig. 7, the $\mathrm{I}_{\text {pol }}$ images between real face, photo paper and iPad display do not give significant differences under both visible and NIR lights. To further confirm the assumption that the $I_{p o l}$ images of each material are relatively similar, the images are statistically analysed. The results show 0 value for the mean, standard deviation, skewness and kurtosis of the $\mathrm{I}_{\mathrm{pol}}$ for the three materials under NIR light. Because of the 0 value, the $\mathrm{I}_{\text {pol }}$ images under NIR light have been omitted from further analysis. Meanwhile, the $\mathrm{I}_{\mathrm{pol}}$ under visible light for all the materials consist small statistical values as shown in Fig. 8(a).

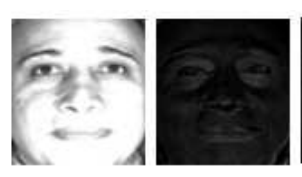

$\mathrm{S}_{0}$

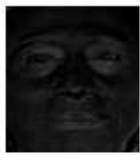

$\mathrm{S}_{2}$

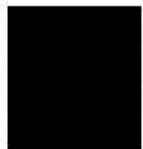

$\mathrm{I}_{\mathrm{pol}}$

Real face

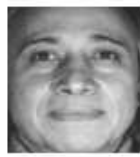

$\mathrm{S}_{0}$

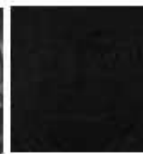

$\mathrm{S}_{1}$

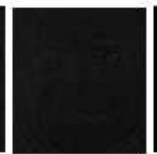

$\mathrm{S}_{2}$

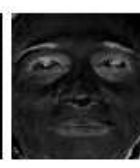

$\mathrm{I}_{\mathrm{SDOLP}}$

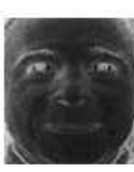

$\mathrm{I}_{\mathrm{SDOLP}}$
Printed photo paper
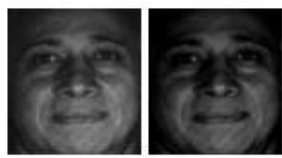

$\mathrm{S}_{0}$

$\mathrm{S}_{1}$
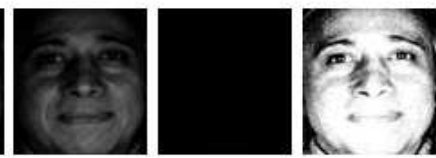

$\mathrm{I}_{\mathrm{SDOLP}}$

iPad display

Visible light
Table 2: Detection rates for the statistical measures of the $\mathrm{I}_{\text {pol }}$ under visible light

\begin{tabular}{lll}
\hline & Percentage (\%) & \\
& - & \\
& TPR & FPR \\
\hline Mean & 91.9 & 8.9 \\
Standard deviation & 89.2 & 6.7 \\
Skewness & 86.5 & 2.2 \\
Kurtosis & 81.1 & 4.4 \\
\hline
\end{tabular}

Table 3: Detection rates of the $\mathrm{I}_{\mathrm{SDOLP}}$ under visible light

\begin{tabular}{lll} 
& Percentage (\%) & \\
& - & \\
& TPR & FPR \\
\hline Mean & 78.4 & 2.2 \\
Standard deviation & 97.3 & 1.1 \\
\hline
\end{tabular}

Table 4: Detection rates between the $\mathrm{I}_{\mathrm{SDOLP}}$ faces and $\mathrm{iPad}$ under NIR light

\begin{tabular}{lll}
\hline & In percentage (\%) & \\
& --- & \\
& TPR & FPR \\
\hline Mean & 87.5 & 4.2 \\
Standard deviation & 87.5 & 0.0 \\
\hline
\end{tabular}

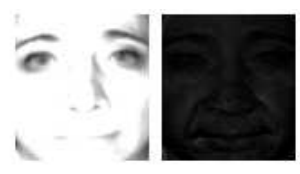

$\mathrm{S}_{0}$
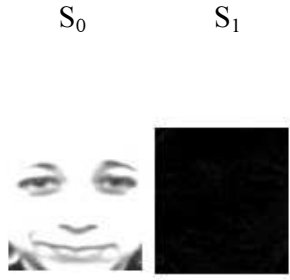

$\mathrm{S}_{0}$

$\mathrm{S}_{1}$

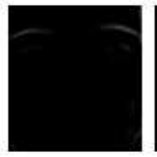

$\mathrm{S}_{2}$

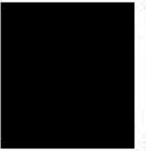

$\mathrm{I}_{\mathrm{pol}}$

Real face

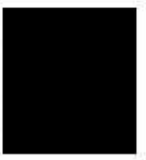

$\mathrm{S}_{2}$

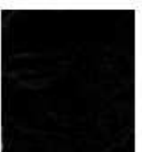

$\mathrm{I}_{\mathrm{pol}}$

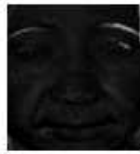

$\mathrm{I}_{\mathrm{SDOLP}}$

Printed photo paper
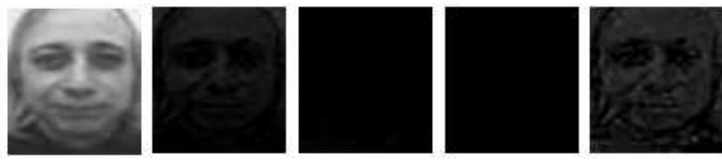

$\mathrm{S}_{0}$

$\mathrm{S}_{1}$

$\mathrm{S}_{2}$

$\mathrm{I}_{\mathrm{pol}}$

$\mathrm{I}_{\mathrm{SDOLP}}$

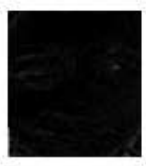

$\mathrm{I}_{\mathrm{SDOLP}}$
iPad display

NIR light

Fig. 7: The Stokes parameters: I, Q and U; the polarization image, $I_{p o l}$ and the Stokes degree of linear polarization 


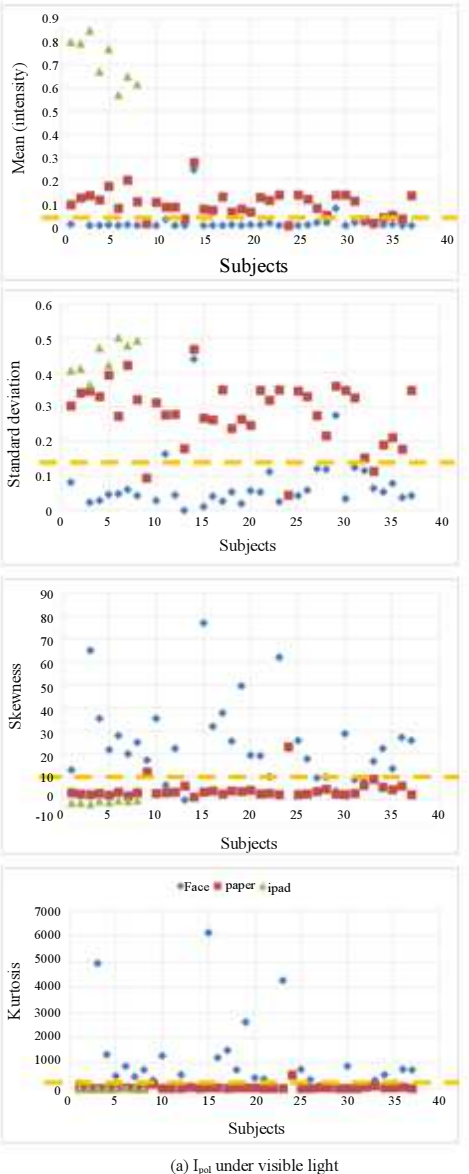

\section{- Real face 1 Photo paper $\triangle$ iPad display}
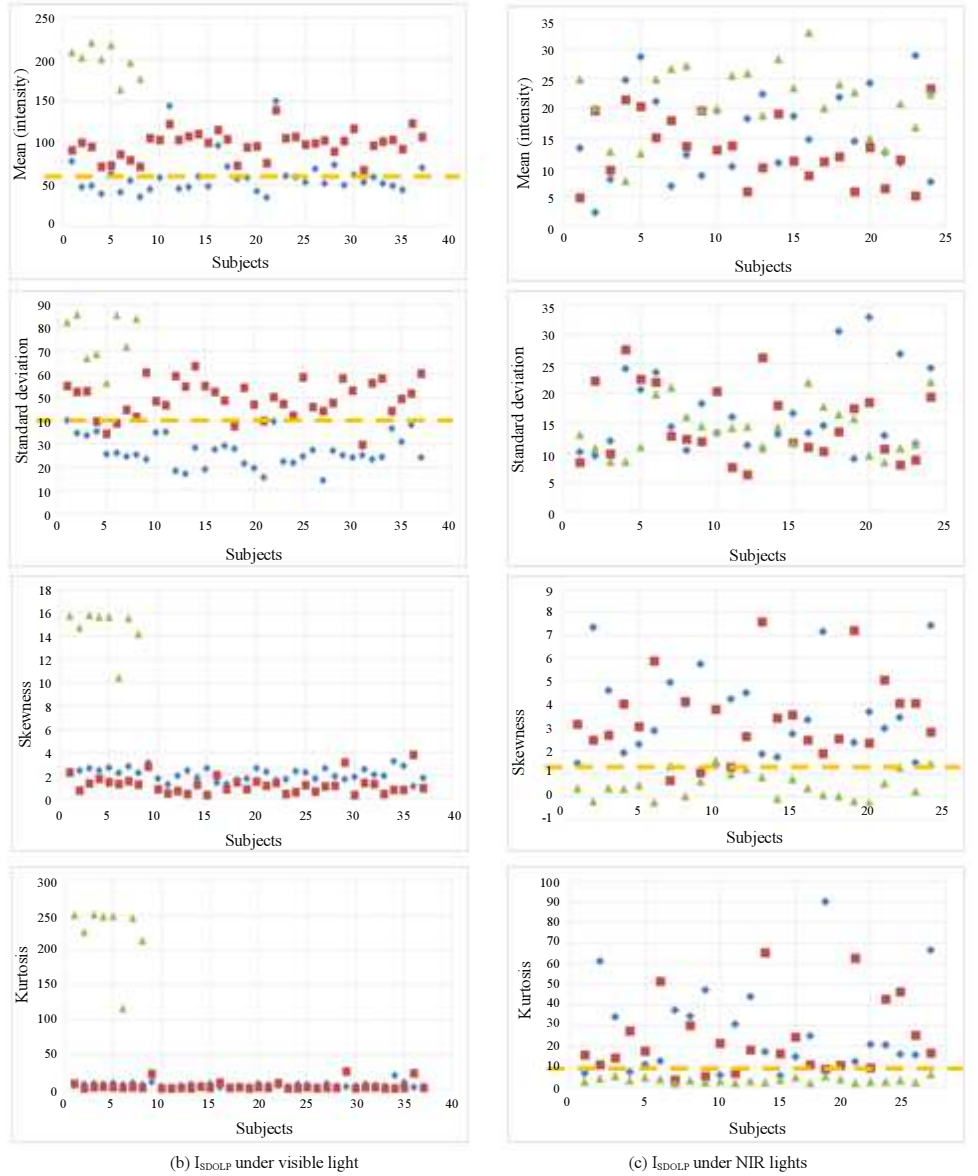

Fig. 8: The mean, standard deviation, skewness and kurtosis scores for (a) the $I_{\text {pol }}$ under visible light; (b) the $I_{S D O L P}$ under visible light; and (c) the $\mathrm{I}_{\mathrm{SDOLP}}$ under NIR lights

From the plots in Fig. 8(a), it can be seen that the differences of the four measurements values between the real faces and the fake traits are quite significant. A threshold value which is represented by the dotted line, is selected for each measure. The $I_{p o l}$ is identified as real face if the mean and standard deviation values are less than the threshold. In addition, the skewness and kurtosis values are more than the corresponding threshold if the $\mathrm{I}_{\text {pol }}$ image is a genuine human face. The detection rates are presented in Table 2.

The $\mathrm{I}_{\mathrm{SDOLP}}$ images in Fig. 7 between real face, photo paper and iPad display under visible light source show clear differences among each other. The statistical scores are plotted in Fig. 8(b). It is clearly shown in Fig. 8(b) that all scores of the $\mathrm{I}_{\mathrm{SDOLP}}$ for iPad display images are far different than the genuine faces.

Apart from that, there are slightly differences of the mean and the standard deviation scores between real faces and paper photo. However, the values for the skewness and the kurtosis between the two materials are relatively similar. Based on these plots, a threshold value is determined only for the mean and the standard deviation scores between genuine faces and printed paper photo. The thresholds are represented by the dotted lines. The $\mathrm{I}_{\text {SDOLP }}$ is classified as a real face if the mean or the standard deviation value is less than the corresponding threshold. The detection rates are listed in Table 3.

Turning now to the $\mathrm{I}_{\mathrm{SDOLP}}$ under NIR light. As can be seen in Fig. 7, there are slight differences between the $\mathrm{I}_{\mathrm{SDOLP}}$ images of the three materials. The statistical measures of the $\mathrm{I}_{\mathrm{SDOLP}}$ under NIR light are plotted in Fig. 8(c). As can be seen from the figure, the four measures do not display any clear threshold between real faces and printed photos. Thus, the $\mathrm{I}_{\mathrm{SDOLP}}$ of the real faces and printed photos under NIR light are statistically indistinguishable. However, there is a rather convincing threshold for the skewness and kurtosis scores between $\mathrm{I}_{\mathrm{SDOLP}}$ real faces and iPad displays. A 
threshold value is selected each for the skewness and kurtosis as indicated by the dotted lines. The $\mathrm{I}_{\mathrm{SDOLP}}$ is classified as real face if the skewness and kurtosis values are more than the corresponding thresholds. Table 4 shows the detection rates for the $\mathrm{I}_{\mathrm{SDOLP}}$ under NIR light between real faces and iPad displays.

\subsection{Detection Using the Bimodality Coefficient}

Beside the statistical analysis, the differences in the shape of data distributions between the real and the fake faces could be applied as another detection parameter. Since the $\mathrm{I}_{\text {pol }}$ images under both visible and NIR light have very low intensity values, PDF distribution could not be computed. Thus, no further detection parameter is carried out on the $\mathrm{I}_{\mathrm{pol}}$.

To further analyse the modality of the distribution, a Probability Density Function (PDF) of each $\mathrm{I}_{\text {SDOLP }}$ image is plotted. Figure 9 presents the PDF plot for the ISDOLP of the three subjects under both visible and NIR lights. As a coarse comparison, the findings from Fig. 9 suggest that in general under the visible light, real face has sharp unimodal shape with relatively thin tail skewed to the right. In contrast, photo paper has more than one peak with wider deviation while iPad attack shows left skewness with single peak on the right side of the distribution.

The PDF plots for the real face and photo paper under the NIR light show similar unimodal shapes while the PDF plot for the iPad attack illustrates multimodality. The modality differences between real face and iPad display could be used as detection parameter. To determine the modality of the ISDOLP distributions, the Bimodality Coefficient (BC) algorithm in Equation (7) is used. The results are summarised in Table 5. From the data in Table 5, it can be seen that 33 out of 37 genuine faces have been identified as having unimodal data distributions under visible light. In contrast, only 9 of the real faces indicates unimodal distributions under NIR light. The similar identification occurs for the photo paper in which most of them indicate unimodal distributions under visible light. However, under NIR light, majority of the photo paper show multimodal distributions.

Table 5: The modality of the $\mathrm{I}_{\mathrm{SDOLP}}$ data distribution using the $\mathrm{BC}$ algorithm

\begin{tabular}{|c|c|c|c|c|}
\hline \multirow[b]{3}{*}{ Modality } & \multicolumn{4}{|c|}{ (Number of subjects) } \\
\hline & \multicolumn{2}{|c|}{ Visible light } & \multicolumn{2}{|c|}{ NIR light } \\
\hline & uni & multi & uni & multi \\
\hline Real face & 33 & 4 & 9 & 15 \\
\hline Photo paper & 22 & 10 & 7 & 17 \\
\hline iPad display & 0 & 8 & 22 & \\
\hline
\end{tabular}

On the other hand, the $\mathrm{BC}$ has classified all the iPad attacks as multimodal distributions under visible light but the modality changed to unimodal under NIR light.

From these results, it can be assumed that it is difficult to differentiate between real face and photo paper by using the $\mathrm{BC}$ alone. However, the $\mathrm{BC}$ gives significant modality distribution differences between real face and the iPad attack under both lights.

\subsection{The Density of Distribution Mode, $m$}

Mode is a number that appears most often in a set of data. In this paper, the mode is the value at the highest peak in each PDF plot as shown in Fig. 9. Since there are unimodal and multimodal distributions, the highest peak of each PDF plot is selected and the density value at the peak is denoted as $\mathrm{m}$. Table 6 compares the range value of $m$ in each PDF plot for the real face, photo paper and iPad attacks under both visible and NIR lights.

From the data in Table 6, there is a clear threshold in $\mathrm{m}$ for the real face and the two attacks under visible light. Based on values, the threshold value of 0.02 has been selected. The image is classified as real face if the $\mathrm{m}$ score is more than 0.02. However, there is no appropriate value could be used as the boundary between genuine face and the fake traits under NIR light. Thus, the density of the distribution mode has been omitted from one of the classification parameters for ISDOLP image captured under NIR light.

\section{The Proposed SDOLP Fast Fusion Formula (SDOLP3F)}

From the results in Section 6, the $I_{\text {pol }}$ images between the real and fake traits under NIR light are indistinguishable. Similarly, the $\mathrm{I}_{\mathrm{SDOLP}}$ between real faces and photo paper under NIR light could not be differentiate. Driven by these results, detection accuracy rates are only calculated for three conditions: (1) $I_{\text {pol }}$ between real and the two fake attacks under visible light; (2) $\mathrm{I}_{\mathrm{SDOLP}}$ between real and the two fake attacks under visible light; and (3) $\mathrm{I}_{\text {SDOLP }}$ between real face and iPad display under NIR light. The summary of the detection accuracy rates is presented in Table 7.

The $I_{\text {pol }}$ and the $I_{S D O L P}$ under both visible and NIR lights have been analysed using six measures: mean, standard deviation, skewness, kurtosis, the $\mathrm{BC}$ and the density of mode. However, not each of the measures give significant detection accuracy rates as can be seen in Table 7. From the results in Table 7 , some of the individual measures present more than $90 \%$ detection accuracy rates for $\mathrm{I}_{\mathrm{pol}}$ and $\mathrm{I}_{\mathrm{SDOLP}}$. To further investigate if higher detection accuracy results could be calculated by fusing some of the measures, these individual measures are transform to algorithm components as: 


$$
\begin{aligned}
& a_{m n}=m n-t h_{m n} \\
& a_{s d}=s d-t h_{s d} \\
& a_{s k}=t h_{s k}-s k \\
& a_{K T}=t h_{k t}-k t \\
& a_{b c}=t h_{b c}-b c \\
& a_{\mathrm{mod}}=t h_{\mathrm{mod}}-\bmod
\end{aligned}
$$

where, $\alpha$ is the algorithm component; $m n$ is the mean; $s d$ is the standard deviation; $s k$ is the skewness; $k t$ is the kurtosis; $b c$ is the bimodality coefficient; mod is the density of mode; and th is the corresponding threshold.

Based on the results presented in Table 7, only two components $\alpha$ with highest detection accuracy rates are selected to be fused in the newly fusion formula known as SDOLP fast fusion formula (SDOLP3F):

$S D O L P 3 F=a_{1} a_{2}$

where, $\alpha_{1}$ and $\alpha_{2}$ are the first and second components in Equation (8) with highest detection rates.

Table 8 presents the results for the SDOLP3F technique. As shown in Table 8 the detection accuracy rate for the $\mathrm{I}_{\mathrm{SDOLP}}$ under visible light increased to 93.9\% compared to the individual measures in Table 7. In addition, the detection accuracy result achieved $89.18 \%$ in the previous study (Zhang et al., 2011) which is less than the achievement of the SDOLP3F. Since the mask databases used in the experiments are different, the comparison on the technique used cannot be made.

\begin{tabular}{|c|c|c|c|c|}
\hline \multirow{3}{*}{ Light sources } & \multirow{2}{*}{\multicolumn{2}{|c|}{\begin{tabular}{l}
$I_{\text {pol }}$ \\
\hdashline Percentage $(\mathbf{\%})$
\end{tabular}}} & \multicolumn{2}{|c|}{$\mathrm{I}_{\mathrm{SDOLP}}$} \\
\hline & & & & \\
\hline & Vis & NIR & Vis & NIR \\
\hline Mean & 91.5 & - & 89.0 & - \\
\hline Standard deviation & 91.5 & - & 92.7 & - \\
\hline Skewness & 92.7 & - & - & 91.7 \\
\hline Kurtosis & 89.0 & - & - & 93.8 \\
\hline The BC & - & - & 63.9 & 77.1 \\
\hline Density of mode & - & - & 90.2 & - \\
\hline
\end{tabular}

Table 6: The $m$ scores of the PDF plots of the $\mathrm{I}_{\mathrm{SDOLP}}$ real face, photo paper and iPad attacks

\begin{tabular}{lll}
\hline & Visible light & NIR light \\
\hline Real face & $0.012-0.48$ & $0.02-0.12$ \\
Photo paper & $0.001-0.19$ & $0.02-0.15$ \\
iPad display & $0.007-0.27$ & $0.02-0.1$ \\
\hline
\end{tabular}

\begin{tabular}{|c|c|c|c|c|}
\hline \multirow[b]{3}{*}{ Light sources } & \multirow{2}{*}{\multicolumn{2}{|c|}{$\begin{array}{l}\mathrm{I}_{\mathrm{pol}} \\
\text { Percentage (\%) }\end{array}$}} & \multicolumn{2}{|c|}{$\mathrm{I}_{\mathrm{SDOLP}}$} \\
\hline & & & & \\
\hline & Vis & NIR & Vis & NIR \\
\hline TPR & 83.8 & - & 97.3 & 83.3 \\
\hline FPR & 6.7 & - & 8.89 & 0 \\
\hline Accuracy rate & 89.0 & - & 93.9 & 91.7 \\
\hline
\end{tabular}

Table 7: The detection accuracy rates for the $\mathrm{I}_{\mathrm{pol}}$ and $\mathrm{I}_{\mathrm{SDOLP}}$ under visible and NIR lights

Table 8: The SDOLP3F detection accuracy rates for the $\mathrm{I}_{\mathrm{pol}}$ and $\mathrm{I}_{\mathrm{SDOLP}}$ images
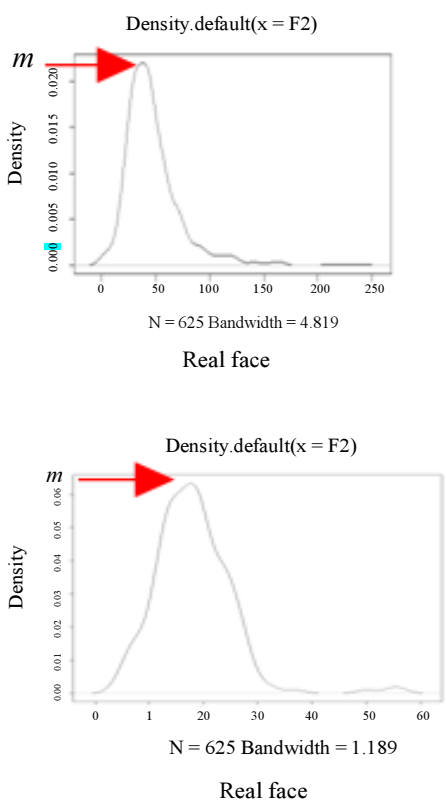
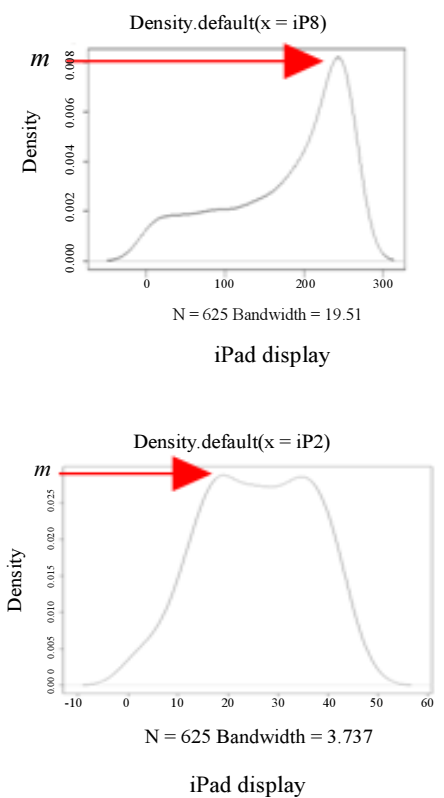

ISDOLP under NIR light

Fig. 9: The probability density (PDF) distribution of the $\mathrm{I}_{\mathrm{SDOLP}}$ for the genuine face, the printed photo paper and the iPad display 
On the other hand, the SDOLP3F results for $\mathrm{I}_{\mathrm{pol}}$ under visible light and $\mathrm{I}_{\mathrm{SDOLP}}$ under NIR light do not achieved higher detection accuracy rates than the individual measures.Taken together, these findings suggest that the SDOLP3F algorithm works well for the $\mathrm{I}_{\mathrm{SDOLP}}$ under visible light and could be the robust face spoofing countermeasure between genuine face, printed photo paper and iPad display based on small sample of subjects.

\section{Conclusion}

From the results presented throughout this paper, there are several conclusions can be made:

- Firstly, there is very small portion of polarized reflected light from the real face, printed photo paper and iPad display under visible light source based on the intensity of the $I_{\text {pol }}$ images as illustrated in Fig. 8

- Secondly, the reflected light from the three surface materials is completely unpolarized under NIR light. The reason behinds these results is that these three materials are classified in same groups which are insulators and opaque objects (LCI, 2017). Thus, the Degree Of Polarization (DOP) between these materials is similar and could not be used as a detection parameter in spite that previous studies applied the DOP to classify materials with different physical properties (Mahendru and Sarkar, 2012; Sarkar et al., 2011)

- Third, the iPad presentation attack under the visible and NIR polarized lights could be distinguish very easily from the genuine face by manipulating the polarization angles. As can be seen from Fig. 6, the iPad polarized image at $90^{\circ}$ polarization angle under both visible and NIR lights are dark compared to the genuine face images at the same angle. The reason could be that the iPad emits its own polarized light which is not permitted to pass through the $\mathrm{P}_{1}$ at the angle of $90^{\circ}$. Thus, any spoofing iPad attack can be successfully prevented at an angle which is perpendicular to the direction of the light wave emitted by the iPad

Real face and photo paper are seen as to have close reflection properties. Both materials produce two types of reflections: surface and subsurface. These have led to the production of relatively similar $\mathrm{I}_{\mathrm{SDOLP}}$. Therefore, most of the data distributions of both materials have been classified as identical to each other. The proposed SDOLP3F fusion technique has shown to be able to differentiate $\mathrm{I}_{\mathrm{SDOLP}}$ between real faces and photo papers based on the intensity variations which caused by the visible polarized reflection. With the classification accuracy of $93.9 \%$, the proposed SDOLP3F algorithm could be appearing as the robust classification algorithm between real face and printed photo.

In contrast, the results do not find any significant differences between $\mathrm{I}_{\text {SDOLP }}$ images of real face and printed photo under NIR polarized light. This is related to the interaction between NIR light and both materials. The NIR radiation penetrates deeper through the human skin and paper (Shao et al., 2010; Mitchell et al., 1945). In addition, NIR can also be absorbed by the deeper human skin and paper properties (Tsuchikawa, 2007; Champagne, 2001). Thus, the intensity of $\mathrm{I}_{\text {SDOLP }}$ face and printed photo images is very low and similar to each other.

For future work, it is suggested to conduct further investigations for a larger sample size. Furthermore, the variety of skin colours could be added into the dataset for the analysis of detection skin colours among subjects.

\section{Acknowledgement}

The authors would like to thank the FassPass and EU FP7 projects for the experimental equipment support particularly to the Computer Vision research group members for participating in the dataset image collection.

\section{Author's Contributions}

Dr. Azim Zaliha Abd Aziz: Main author collecting data, run the experiments and write the manuscripts.

\section{Ethics}

The Author declare that there are no ethical issues associated with this work.

\section{References}

Abd, A., H. Wei and J. Ferryman, 2017. Face Antispoofing countermeasure: Efficient 2D materials classification using polarization imaging. Proceedings of the 5th International Workshop on Biometrics and Forensics, Apr. 4-5, IEEE Xplore Press, Coventry, UK. DOI: 10.1109/IWBF.2017.7935105

Bae, E.J., S.H. Seo, Y.C. Kye and H.H. Ahn, 2010. A quantitative assessment of the human skin surface using polarized light digital photography and its dermatologic significance. Skin Res. Technol., 16: 270-274. DOI: 10.1111/j.1600-0846.2010.00439.x

Bai, J., N.T. Tsong, G. Xinting and S. Yun-Qing, 2010. Is physics-based liveness detection truly possible with a single image? Proceedings of the IEEE International Symposium on Circuits and Systems, May 2, IEEE Xplore Press, Paris, France, pp: 3425-3428. DOI: 10.1109/ISCAS.2010.5537866 
Bin, Z., J. Xuan, H. Zhao, G.J. Chepko and M.T. Freedman et al., 2007. Polarization imaging for breast cancer diagnosis using texture analysis and SVM. Proceedings of the IEEE/NIH Life Science Systems and Applications Workshop, Nov. 8-9, IEEE Xplore Press, Bethesda, MD, USA, pp: 217-220. DOI: 10.1109/LSSA.2007.4400923

Champagne, M., 2001. The use of orthogonal signal correction to improve NIR readings of pulp fibre properties. Pulp paper Canada, 102: 41-43.

Erdogmus, N. and S. Marcel, 2013. Spoofing in 2D face recognition with $3 \mathrm{D}$ masks and anti-spoofing with Kinect. Proceedings of the IEEE 16th International Conference on Biometrics: Theory, Applications and Systems, Sept. 2, IEEE Xplore Press, Arlington, VA, USA, pp: 1-6. DOI: 10.1109/BTAS.2013.6712688

Hua, C. and L.B. Wolff, 1996. Polarization phase-based method for material classification and object recognition in computer vision. Proceedings of the CVPR IEEE Computer Society Conference on Computer Vision and Pattern Recognition, Jun. 1820, IEEE Xplore Press, San Francisco, CA, USA, pp: 128-135. DOI: 10.1109/CVPR.1996.517064

Jacques, S.L., J.R. Roman and K. Lee, 2000. Imaging superficial tissues with polarized light. Lasers surgery medi., 26: 119-129.

Jain, A.K., A. Ross and S. Prabhakar, 2004. An introduction to biometric recognition. Transact. Circuits Syst. Video Technol., 14: 4-20.

DOI: 10.1109/TCSVT.2003.818349

Jukka, M., H. Abdenour and M. Pietikäinen, 2011. Face spoofing detection from single images using microtexture analysis. Proceedings of the IEEE Biometrics Compendium IEEE RFIC Virtual Journal IEEE, RFID Virtual Journal, Oct. 11-13, IEEE Xplore Press, Washington, DC, USA, pp: 1-7. DOI: 10.1109/IJCB.2011.6117510

Kim, Y., J. Na, S. Yoon and J. Yi, 2009. Masked fake face detection using radiance measurements. J. Optical Soci. Am. A., 26: 760-766.

DOI: $10.1364 /$ JOSAA.26.000760

Kose, N. and J.L. Dugelay, 2013a. Reflectance analysis based countermeasure technique to detect face mask attacks. Proceedings of the 18th International Conference on Digital Signal Processing (DSP), Jul. 1-3, IEEE Xplore Press, Fira, Greece, pp: 1-6. DOI: 10.1109/ICDSP.2013.6622704

Kose, N. and J.L. Dugelay, 2013b. Countermeasure for the protection of face recognition systems against mask attacks. Proceedings of the 10th IEEE International Conference and Workshops on Automatic Face and Gesture Recognition (FG), Apr., 22-26, IEEE Xplore Press, Shanghai, China, pp: 1-6. DOI: 10.1109/FG.2013.6553761
LCI, 2017. https://schoolworkhelper.net/list-ofconductors-and-insulators/

Li, J., W. Yunhong, T.T. Jain and K. Anil, 2004. Live face detection based on the analysis of fourier spectra. Defense Secur., 5404: 296-303. DOI: $10.1117 / 12.541955$

Mahendru, A. and M. Sarkar, 2012. Bio-inspired object classification using polarization imaging. Proceedings of the 16th International Conference on Sensing Technology, Dec. 18-21, IEEE Xplore Press, Kolkata, India, pp: 207-212. DOI: 10.1109/ICSensT.2012.6461672

Matsubara, A., 2012. Differences in the surface and subsurface reflection characteristics of facial skin by age group. Skin Res. Technol., 18: 29-35. DOI: $10.1111 / \mathrm{j} .1600-0846.2011 .00537 . x$

Mitchell, H., T.S. Hamilton, F.R. Steggerda and H.W. Bean, 1945. The chemical composition of the adult human body and its bearing on the biochemistry of growth. J. Bio. Chem., 168: 625-637.

Pan, G., L. Sun and Z. Wu, 2008. Liveness detection for face recognition. INTECH Open Access Publisher.

Pfister, R., K.A. Schwarz, M. Janczyk, R. Dale and J.B. Freeman, 2013. Good things peak in pairs: A note on the bimodality coefficient. Front. Psychol., 4: 1-700. DOI: 10.3389/fpsyg.2013.00700

Philp, J., N. Carter and C.P. Lenn,1987. Improved optical discrimination of skin with polarized light. J. Soci. Cosmetic Chemists., 39: 121-132.

Picture of the skin, 2015. http://www.webmd.com/skinproblems-andtreatments/picture-of-the-skin

Rudd, E.M., M. Günther and T.E. Boult, 2016. PARAPH: Presentation attack rejection by analyzing polarization hypotheses. Proceedings of the IEEE Conference on Computer Vision and Pattern Recognition Workshops, Jun. 26, IEEE Xplore Press, Las Vegas, NV, USA, pp: 171-178. DOI: 10.1109/CVPRW.2016.28

Sarkar, M., S. David, S.S.S. Bello, C. Hoof and A. Theuwissen, 2011. Integrated polarization analyzing CMOS image sensor for material classification. IEEE Sensors J., 11: 1692-1703. DOI: 10.1109/JSEN.2010.2095003

Shao, X., W. Zheng and Z. Huang, 2010. Polarized near-infrared autofluorescence imaging combined with near-infrared diffuse reflectance imaging for improving colonic cancer detection. Optics Express, 18: 24293-24300. DOI: $10.1364 / O E .18 .024293$

So-Ling, C. and L. Ling, 2001. A multi-layered reflection model of natural human skin. Proceedings of the Computer Graphics International, Jul. 6-6, IEEE Xplore Press, Hong Kong, China, pp: 249-256. DOI: $10.1109 /$ CGI.2001.934681 
Tsuchikawa, S., 2007. A review of recent near infrared research for wood and paper. Applied Spectroscopy Rev., 42: 43-71.

Wolff, L.B. and T.E. Boult, 1989. Polarization/radiometric based material classification. Proceedings of the IEEE Computer Society Conference on Computer Vision and Pattern Recognition, Jun. 4-8, IEEE Xplore Press, San Diego, CA, USA, pp: 387-395. DOI: $10.1109 /$ CVPR.1989.37876

Wolff, L.B., 1990. Polarization-based material classification from specular reflection. IEEE Transact. Pattern Analysis Machine Intelligence, 12: 1059-1071. DOI: 10.1109/34.61705

Wolff, L.B., 1989. Using polarization to separate reflection components. Proceedings of the CVPR 89: IEEE Computer Society Conference on Computer Vision and Pattern Recognition, Jun. 48, IEEE Xplore Press, San Diego, CA, USA, pp: 363-369. DOI: 10.1109/CVPR.1989.37873
Zallat, J., P. Grabbling and Y. Takakura, 2003. Using polarimetric imaging for material classification. Proceedings of the International Conference on Image Processing, Sept. 14-17, IEEE Xplore Press, Barcelona, Spain, pp: 827-30. DOI: 10.1109/ICIP.2003.1246808

Zhang, Z., D. Yi, Z. Lei and S.Z. Li, 2011. Face liveness detection by learning multispectral reflectance distributions. Proceedings of the IEEE International Conference on Face and Gesture, Mar. 21-25, IEEE Xplore Press, Santa Barbara, CA, USA, pp: 436-441. DOI: $10.1109 /$ FG.2011.5771438 